\title{
ERRATUM: "STELLAR KINEMATICS AND STRUCTURAL PROPERTIES OF VIRGO CLUSTER DWARF EARLY-TYPE GALAXIES FROM THE SMAKCED PROJECT. II. THE SURVEY AND A SYSTEMATIC ANALYSIS OF KINEMATIC ANOMALIES AND ASYMMETRIES” (2014, APJS, 215, 17)
}

\author{
E. Toloba ${ }^{1,2}$, P. Guhathakurta ${ }^{1}$, R. F. Peletier ${ }^{3}$, A. Boselli ${ }^{4}$, T. Lisker ${ }^{5}$, J. Falcón-Barroso ${ }^{6,7}$, J. D. Simon ${ }^{2}$, \\ G. van de $\mathrm{VeN}^{8}$, S. PAudel ${ }^{9}$, E. Emsellem ${ }^{10,11}$, J. JANZ ${ }^{12}$, M. DEN BroK ${ }^{13}$, J. Gorgas ${ }^{14}$, G. Hensler ${ }^{15}$, E. LauriKainen ${ }^{16,17}$, \\ S.-M NIEMI ${ }^{18}$, A. RYśs ${ }^{6,7}$, AND H. SALO ${ }^{16}$ \\ ${ }^{1}$ UCO/Lick Observatory, University of California, Santa Cruz, 1156 High Street, Santa Cruz, CA 95064, USA; toloba@ ucolick.org \\ ${ }^{2}$ Observatories of the Carnegie Institution for Science, 813 Santa Barbara Street, Pasadena, CA 91101, USA; \\ ${ }^{3}$ Kapteyn Astronomical Institute, Postbus 800, 9700 AV Groningen, The Netherlands \\ ${ }^{4}$ Laboratoire d'Astrophysique de Marseille-LAM, Université d'Aix-Marseille \& CNRS, UMR 7326, 38 rue F. Joliot-Curie, 13388 Marseille Cedex 13, France \\ ${ }^{5}$ Astronomisches Rechen-Institut, Zentrum für Astronomie der Universität Heidelberg, Mönchhofstraße 12-14, D-69120 Heidelberg, Germany \\ ${ }^{6}$ Instituto de Astrofísica de Canarias, Vía Láctea s/n, La Laguna, Tenerife, Spain \\ ${ }^{7}$ Departamento de Astrofísica, Universidad de La Laguna, E-38205, La Laguna, Tenerife, Spain \\ ${ }^{8}$ Max Planck Institute for Astronomy, Königstuhl 17, 69117 Heidelberg, Germany \\ ${ }^{9}$ Korea Astronomy and Space Science Institute, Daejeon 305-348, Korea \\ ${ }^{10}$ European Southern Observatory, Karl-Schwarzschild-Str. 2, 85748, Garching, Germany \\ ${ }^{11}$ Université Lyon 1, Observatoire de Lyon, Centre de Recherche Astrophysique de Lyon and Ecole Normale Supérieure de Lyon, 9 Avenue Charles André, F-69230, \\ Saint-Genis Laval, France \\ ${ }^{12}$ Centre for Astrophysics and Supercomputing, Swinburne University, Hawthorn, VIC 3122, Australia \\ ${ }^{13}$ Department of Physics and Astronomy, University of Utah, Salt Lake City, UT 84112, USA \\ ${ }^{14}$ Departamento de Astrofísica y Física de la Atmósfera, Universidad Complutense de Madrid, 28040, Madrid, Spain
${ }_{15}$, \\ ${ }^{15}$ University of Vienna, Department of Astrophysics, Türkenschanzstraße 17, 1180 Vienna, Austria \\ ${ }^{16}$ Division of Astronomy, Department of Physics, PO Box 3000, FI-90014 University of Oulu, Finland \\ ${ }^{17}$ Finnish Center for Astronomy with ESO (FINCA), University of Turku, Finland \\ ${ }^{18}$ Mullard Space Science Laboratory, University College London, Holmbury St. Mary, Dorking, Surrey RH5 6NT, UK \\ Received 2015 January 15; accepted 2015 January 21; published 2015 March 24
}

In the published article Toloba et al. (2014), Column 3 of Table 8 appears in ascending order. Thus the stellar mass values do not correspond to the galaxy indicated in Column 1 of the same Table. Only Column 3 is affected by this effect, no other Column in Table 8 or any other Table is affected, as well as any Figure or any derived quantity. This typographic mistake does not affect our results and conclusions. We provide below the corrected Table. 
Table 1

Masses and Dark Matter Fractions

\begin{tabular}{|c|c|c|c|c|c|}
\hline $\begin{array}{l}\text { Galaxy } \\
\text { (1) }\end{array}$ & $\begin{array}{c}\log M_{e} \\
M_{\odot} \\
(2)\end{array}$ & $\begin{array}{c}\log M_{e}^{*} \\
M_{\odot} \\
(3)\end{array}$ & $\begin{array}{c}f_{\mathrm{DM}} \\
(4)\end{array}$ & $\begin{array}{c}(M / L)_{d y n, r} \\
M_{\odot} / L_{\odot, r} \\
\quad(5)\end{array}$ & $\begin{array}{c}(M / L)_{d y n, H} \\
M_{\odot} / M_{\odot, H} \\
\quad(6)\end{array}$ \\
\hline VCC0009 & $9.24 \pm 0.14$ & $9.10 \pm 0.12$ & $0.28 \pm 0.30$ & $2.62 \pm 0.83$ & $0.67 \pm 0.21$ \\
\hline VCC0021 & $8.88 \pm 0.11$ & $8.48 \pm 0.12$ & $0.61 \pm 0.15$ & $3.19 \pm 0.81$ & $1.22 \pm 0.31$ \\
\hline VCC0033 & $8.56 \pm 0.19$ & $8.47 \pm 0.12$ & $0.19 \pm 0.41$ & $1.82 \pm 0.78$ & $0.59 \pm 0.25$ \\
\hline VCC0170 & $9.11 \pm 0.15$ & $8.75 \pm 0.12$ & $0.56 \pm 0.20$ & $3.16 \pm 1.08$ & $1.08 \pm 0.37$ \\
\hline VCC0308 & $8.93 \pm 0.12$ & $8.94 \pm 0.12$ & $-0.03 \pm 0.40$ & $1.53 \pm 0.42$ & $0.47 \pm 0.13$ \\
\hline VCC0389 & $9.04 \pm 0.09$ & $9.00 \pm 0.12$ & $0.08 \pm 0.31$ & $1.82 \pm 0.36$ & $0.52 \pm 0.10$ \\
\hline VCC0397 & $9.02 \pm 0.08$ & $8.57 \pm 0.12$ & $0.64 \pm 0.12$ & $5.73 \pm 1.08$ & $1.35 \pm 0.25$ \\
\hline VCC0437 & $9.42 \pm 0.10$ & $8.96 \pm 0.12$ & $0.65 \pm 0.13$ & $4.54 \pm 1.02$ & $1.37 \pm 0.31$ \\
\hline VCC0523 & $9.31 \pm 0.07$ & $9.11 \pm 0.12$ & $0.37 \pm 0.20$ & $1.98 \pm 0.30$ & $0.76 \pm 0.11$ \\
\hline VCC0543 & $9.16 \pm 0.08$ & $8.84 \pm 0.12$ & $0.52 \pm 0.16$ & $3.05 \pm 0.56$ & $1.00 \pm 0.19$ \\
\hline VCC0634 & $9.15 \pm 0.09$ & $8.96 \pm 0.12$ & $0.36 \pm 0.22$ & $1.66 \pm 0.34$ & $0.75 \pm 0.15$ \\
\hline VCC0750 & $9.26 \pm 0.08$ & $8.50 \pm 0.12$ & $0.83 \pm 0.06$ & $8.35 \pm 1.52$ & $2.78 \pm 0.51$ \\
\hline VCC0751 & $8.83 \pm 0.10$ & $8.75 \pm 0.12$ & $0.17 \pm 0.29$ & $1.97 \pm 0.43$ & $0.58 \pm 0.13$ \\
\hline VCC0781 & $9.09 \pm 0.09$ & $8.66 \pm 0.12$ & $0.63 \pm 0.13$ & $4.36 \pm 0.87$ & $1.29 \pm 0.26$ \\
\hline VCC0794 & $9.15 \pm 0.13$ & $8.47 \pm 0.12$ & $0.79 \pm 0.08$ & $4.73 \pm 1.37$ & $2.30 \pm 0.66$ \\
\hline VCC0856 & $9.01 \pm 0.12$ & $8.84 \pm 0.12$ & $0.33 \pm 0.26$ & $2.27 \pm 0.64$ & $0.72 \pm 0.20$ \\
\hline VCC0917 & $8.75 \pm 0.09$ & $8.37 \pm 0.12$ & $0.58 \pm 0.15$ & $3.70 \pm 0.80$ & $1.15 \pm 0.25$ \\
\hline VCC0940 & $9.30 \pm 0.08$ & $8.71 \pm 0.12$ & $0.74 \pm 0.09$ & $6.32 \pm 1.16$ & $1.85 \pm 0.34$ \\
\hline VCC0990 & $8.99 \pm 0.07$ & $8.74 \pm 0.12$ & $0.43 \pm 0.18$ & $2.87 \pm 0.47$ & $0.85 \pm 0.14$ \\
\hline VCC1010 & $9.33 \pm 0.07$ & $9.17 \pm 0.12$ & $0.29 \pm 0.23$ & $2.57 \pm 0.41$ & $0.68 \pm 0.11$ \\
\hline VCC1087 & $9.26 \pm 0.07$ & $8.99 \pm 0.12$ & $0.46 \pm 0.17$ & $1.82 \pm 0.29$ & $0.88 \pm 0.14$ \\
\hline VCC1122 & $9.01 \pm 0.09$ & $8.62 \pm 0.12$ & $0.59 \pm 0.14$ & $3.81 \pm 0.76$ & $1.18 \pm 0.24$ \\
\hline VCC1183 & $9.33 \pm 0.07$ & $8.89 \pm 0.12$ & $0.64 \pm 0.12$ & $4.27 \pm 0.71$ & $1.33 \pm 0.22$ \\
\hline VCC1261 & $9.41 \pm 0.06$ & $9.18 \pm 0.12$ & $0.42 \pm 0.18$ & $2.85 \pm 0.43$ & $0.82 \pm 0.12$ \\
\hline VCC1304 & $8.81 \pm 0.12$ & $8.52 \pm 0.12$ & $0.48 \pm 0.20$ & $3.13 \pm 0.84$ & $0.92 \pm 0.25$ \\
\hline VCC1355 & $8.89 \pm 0.18$ & $8.72 \pm 0.12$ & $0.32 \pm 0.34$ & $1.90 \pm 0.80$ & $0.71 \pm 0.30$ \\
\hline VCC1407 & $8.93 \pm 0.09$ & $8.59 \pm 0.12$ & $0.54 \pm 0.16$ & $3.74 \pm 0.80$ & $1.04 \pm 0.22$ \\
\hline VCC1431 & $9.20 \pm 0.06$ & $8.93 \pm 0.12$ & $0.46 \pm 0.17$ & $3.49 \pm 0.47$ & $0.89 \pm 0.12$ \\
\hline VCC1453 & $9.15 \pm 0.08$ & $8.94 \pm 0.12$ & $0.40 \pm 0.20$ & $2.72 \pm 0.49$ & $0.79 \pm 0.14$ \\
\hline VCC1528 & $9.05 \pm 0.06$ & $8.79 \pm 0.12$ & $0.46 \pm 0.17$ & $3.22 \pm 0.47$ & $0.88 \pm 0.13$ \\
\hline VCC1549 & $9.01 \pm 0.08$ & $8.78 \pm 0.12$ & $0.42 \pm 0.20$ & $3.53 \pm 0.68$ & $0.83 \pm 0.16$ \\
\hline VCC1684 & $8.85 \pm 0.09$ & $8.34 \pm 0.12$ & $0.69 \pm 0.11$ & $4.24 \pm 0.88$ & $1.54 \pm 0.32$ \\
\hline VCC1695 & $8.84 \pm 0.11$ & $8.71 \pm 0.12$ & $0.27 \pm 0.28$ & $1.67 \pm 0.44$ & $0.66 \pm 0.17$ \\
\hline VCC1861 & $9.06 \pm 0.09$ & $8.89 \pm 0.12$ & $0.33 \pm 0.23$ & $2.17 \pm 0.44$ & $0.72 \pm 0.14$ \\
\hline VCC1895 & $8.73 \pm 0.13$ & $8.52 \pm 0.12$ & $0.38 \pm 0.25$ & $2.41 \pm 0.72$ & $0.78 \pm 0.23$ \\
\hline VCC1910 & $9.03 \pm 0.07$ & $9.03 \pm 0.12$ & $0.00 \pm 0.33$ & $2.05 \pm 0.35$ & $0.48 \pm 0.08$ \\
\hline VCC1912 & $9.23 \pm 0.08$ & $8.91 \pm 0.12$ & $0.52 \pm 0.16$ & $3.36 \pm 0.60$ & $0.99 \pm 0.18$ \\
\hline VCC1947 & $9.11 \pm 0.06$ & $8.91 \pm 0.12$ & $0.36 \pm 0.20$ & $3.19 \pm 0.44$ & $0.75 \pm 0.10$ \\
\hline VCC2083 & $8.93 \pm 0.10$ & $8.27 \pm 0.12$ & $0.78 \pm 0.08$ & $6.51 \pm 1.55$ & $2.17 \pm 0.52$ \\
\hline
\end{tabular}

Note. Column 1: galaxy name. Column 2: dynamical mass within the $R_{e}$ estimated as described in Equation 6 of Toloba et al. (2014). Column 3: stellar mass within the $R_{e}$ estimated assuming a stellar mass-to-light ratio of $(M / L)_{H}^{*}=0.73 \pm 0.19$ for all dEs. The average mass does not change if we assume a different $(M / L)_{H}^{*}$ or $(M / L)_{V}^{*}$ for each dE (see Section 9 of Toloba et al. (2014)). The total dynamical masses and the total stellar masses can be calculated by multiplying by 2 the masses in columns 2 and 3. Column 4: dark matter fraction within the $R_{e}$ estimated as described in Equation 9 of Toloba et al. (2014). Note that negative values of $f_{\mathrm{DM}}$ are consistent with no dark matter within the uncertainties. Columns 5 and 6: dynamical mass-to-light ratio calculated dividing the dynamical masses in Column 1 by half the luminosities obtained from the $r$ and $H$ band absolute magnitudes in Table 4 of Toloba et al. (2014), respectively.

\section{REFERENCES}

Toloba, E., Guhathakurta, P., Peletier, R. F., et al. 2014, ApJS, 215, 17 